\title{
Use of Biomarkers in Individualization of Treatment
}

\author{
Presented by Jennifer J.D. Morrissette, PhD
}

\begin{abstract}
Next-generation sequencing (NGS), also known as massively parallel sequencing (MPS), offers broad detection of genetic alterations that, in approximately one-third of patients with cancer, are "actionable," meaning that they can be targeted by available therapeutics or the detection of the alteration can lead to a change in therapy. NGS is useful in the diagnosis of patients, determining their prognosis, appropriate treatment selection, and clinical trial enrollment. Many testing panels are available, each with different abilities to detect various mutation types. Clinicians not only have to decide which test to use, but which specimen to test, and when and how often to test. Aside from unique mutations, immunotherapy markers have become important for the use of checkpoint inhibitors, and their detection and interpretation can also be challenging. Efforts are underway to simplify and validate these assays. Meanwhile, clinicians should become educated about the benefit of, means of, and interpretation of genomic testing patients across the disease course.
\end{abstract}

J Natl Compr Canc Netw 2020;18(7.5):989-991 doi: 10.6004/jnccn.2020.5007

Next-generation sequencing (NGS) is a technique for profiling genetic and genomic changes that can be associated with disease and may be diagnostic or prognostic or may alter treatment. "It's a difficult field. Education regarding how results are generated and how to use them will be the most important aspect in getting the most out of genomic testing in treating your patients," Jennifer J.D. Morrissette, $\mathrm{PhD}$, Associate Professor, Department of Clinical Pathology and Laboratory Medicine, and Laboratory Director of the CytoGenomics Laboratory, Abramson Cancer Center at the University of Pennsylvania, said in her presentation at the NCCN 2020 Virtual Annual Conference.

Dependant on the assay design, NGS commonly identifies the following types of alterations: (1) base pair substitutions, including single nucleotide variants (SNVs) (eg, KRAS G12C); (2) insertions and deletions, including indels, typically up to 70 base pairs (eg, EGFR E746_A750del); (3) copy number gains and losses, including amplifications and deletions (eg, ERBB2 CNG); and (4) translocations and rearrangements, including interchromosomal or intrachromosomal rearrangements (eg, ETV6-NTRK3 fusion, a pan-cancer genomic change). A user-friendly reference for the interpretation of genomic variants can be found in the Association of Molecular Pathology Pocket Guides. ${ }^{1}$

Genetic testing in oncology has evolved from singlegene or single-site testing, to "hot spot panels," to comprehensive genomic profiling, such as NGS, in 2020. NGS has become broadly adopted, with $>250$ College of
American Pathologists (CAP)-certified academic and commercial laboratories. Dr. Morrissette predicted that the future of testing will be much more complex, including exome and genome analysis, whole transcriptome analysis, comparisons of tumor and tumor microenvironment, genomic-based precancer screening, multiomics, and detection of different types of low-risk variants.

\section{NGS in Oncology}

NGS identifies alterations that can make a difference in diagnosis, especially in some malignancies, such as brain tumors, in addition to determining prognosis and deciphering whether a tumor is primary or metastatic. However, its most important role currently is in identifying tissuespecific targetable mutations and resistance mutations to guide treatment selection. The FDA has a growing list of genomic biomarkers associated with specific drugs in the field of oncology that include targeted therapeutics, contraindications, clinical pharmacology, and clinical studies.

NGS also helps determine patient eligibility for clinical trials, and it detects germline variants that may be informative for screening patients and other family members. Of note, Dr. Morrissette added, $90 \%$ of laboratories test only the tumor and do not perform matched germline testing, based on supplementary material from CAP proficiency surveys. "This means that when a variant is detected, it's not clear whether it's germline or acquired," she cautioned.

\section{Targetable Mutations}

Acquired variants are commonly detected by NGS. A review of 10,000 patients tested using the MSK-IMPACT 
multiplex panel identified at least 1 targetable mutation in $37 \%$ of patients; all of the $>30$ tumor types had at least 1 such alteration, although the level of evidence for targetability varied. ${ }^{2}$ For $\geq 5$ principal malignancies, $97 \%$ of the genes in the panel were mutated at least once, reinforcing the potential benefit of broad mutation profiling regardless of lineage. "The poster child for this is non-small cell lung cancer (NSCLC)," she said.

In lung adenocarcinoma, the variants recommended by the NCCN Guidelines can be detected by NGS testing, such as SNVs/indels, including driver mutations in KRAS, $E G F R, B R A F, M E T$, and PIK3CA. It can also detect the recurrent fusions or rearrangements involving $A L K, R E T$, ROS1, NTRK1, NTRK2, and NTRK3; copy number variations in MET, ERBB2, and EGFR; and complex mutational signatures, including microsatellite instability (MSI) and tumor mutational burden (TMB). Although the detection of these variants is possible with NGS, she cautioned that this depends on "how the laboratory has designed and validated the test regarding whether or not all these variants are reportable or were even captured in the assay design." NCCN recommends broad molecular profiling in NSCLC to reveal these variants while minimizing wastage of tissue.

When tissue is not available, circulating tumor DNA (ctDNA) can monitor treatment response and identify resistance mutations. The amount of ctDNA that is detected in plasma is related to TMB but may not represent all clones. ${ }^{3-5}$ The recommendation is to use ctDNA in conjunction with tissue testing.

\section{Barriers and Challenges to NGS Adoption}

Despite its great utility, there are challenges to the broad adoption of NGS, including preanalytic (specimen) variables, differences in tumor biology and in assays, and issues related to reimbursement.

Barriers associated with preanalytic variables include low tumor cellularity, poor sample quality, and limited availability of tissue. The 3 types of specimensformalin-fixed, paraffin-embedded (FFPE), fresh tissue, and fine-needle aspiration/pleural fluids-also have unique considerations. For example, FFPE specimens can vary in quantity and quality, sometimes yielding insufficient DNA for detecting variants or tumor heterogeneity, or insufficient quality of DNA for the assay. Additionally, quantification of tumor percentage can be complicated, and if inaccurately assessed, can result in submission of tumor that falls below the validated reporting threshold, which can result in a false-negative. "It's complicated, deciding what test to order for your particular tumor type. Decisions are based on tumor type, performance status, and appropriate material after discussions with your pathologist," Dr. Morrissette said, adding that institutional consensus on these factors can a "solution."

\section{Choosing the Right Test}

"There are also barriers in choosing the right test for tumor testing. The question is, what information do you need in order to treat your patient?" Dr. Morrissette continued. Variability is common among the assays and among the contents of their panels in terms of genes and coverage within genes, allele fraction cutoffs, types of variants reported, and cutoff values for the different mutation types.

"Understand the limitations of the NGS assay being ordering," she advised. "Read the fine print so you know which genes of interest will be covered and which types of variants will be reported." The fine print on assays can be informative for the specific genes of interest, types of variants (ie, NTRK mutations vs NTRK fusions, which are not equivalent), tumor enrichment, and the limits of the tests' detection. A low tumor percentage can result in a false-negative or missed detection of heterogeneity. "Knowing what you ordered is important for understanding what result you are getting," she continued. "In addition, considering that another barrier of testing is turn-around time, ask whether the results will be available in time to treat your patient. If not, consider alternative assays, such as single gene stand-alone testing for a mutation that is critical for treating the patient immediately."

To interpret the results, clinicians have additional online resources in addition to the NCCN Biomarkers Compendium. For more complex results, additional support may be needed, such as from the testing laboratory.

\section{Reimbursement Issues}

The large variability in reimbursement for these tests is another barrier. Some payers cover large NGS panels while others rely on the NCCN Biomarkers Compendium for guidance. In addition, coverage policies are complicated and the requirement for preauthorization can further burden clinicians and laboratories. Often, some degree of clinical utility must be demonstrated for reimbursement.

"Spending more on diagnostics has been shown to translate into spending less on therapies that don't work," Dr. Morrissette pointed out, advising attendees to emphasize this point to any insurance company who questions the order. ${ }^{6}$

\section{Using NGS in Selecting Immunotherapy}

With pan-cancer indications now approved for immunotherapy, NGS has another use: identifying certain variant profiles associated with response to checkpoint inhibitors. "Tissue agnostic approvals may mark a new 
chapter for predictive biomarkers, with pembrolizumab the first pan-cancer approval," she said.

Currently, tissue-based testing of PD-L1 by immunohistochemistry yields a tumor proportion score that can be used to stratify patients for treatment. However, PD-L1 is an imperfect biomarker because it is determined by different antibodies and assay, and scoring systems vary by tissue type. Another means is to look for mismatch repair deficiency (dMMR) and MSI (a molecular feature of dMMR), these also serve as pan-cancer biomarkers for response to checkpoint blockade. dMMR and MSI are associated with the accumulation of large numbers of variants and their presence heralds the accumulation of a high number of mutations. MMR/MSI can be assessed by different methods. MMR is evaluated by immunohistochemistry, whereas MSI can be tested using PCR or NGS. Assessment of MSI pan-cancer is often easiest to include MSI testing within routine NGS assays, according to Dr. Morrissette. "dMMR is common in some tumor types (prevalence $>10 \%$ ) and much less in others $(<1 \%)$. Especially where it is less common, combining MSI detection with NGS may be more costeffective than testing for dMMR with immunohistochemistry," she suggested.

The emerging biomarker TMB is a surrogate for the detection of neoantigens, such as tumor-specific antigens that must be recognized for the activation of $\mathrm{T}$ cells. TMB is a quantitative measure of the total number of somatic nonsynonymous mutations per coding area of a tumor genome, and is measured in mutations per megabase (mut/Mb). Panel sequencing of the tumor-the approach most laboratories choose-has been shown to correlate with TMB derived from whole-exome sequencing. ${ }^{7,8}$ Current limitations to using TMB as a biomarker include the inconsistent correlation between $\mathrm{TMB}$ and objective response to treatment, the lack of data harmonization among laboratories, and the variability among tests in the amount of genome they cover and how results are reported, which can confuse interpretation of the results. Other barriers to its implementation are the high variability of neoantigen load among tumor types and the lack of a standardized, clinically validated scale providing optimal cutoffs that define a high TMB predictive of clinical benefit.

Recently, on June 16, 2020, the FDA granted accelerated approval to pembrolizumab for pediatric and adult solid tumors for the treatment of adult and pediatric patients with unresectable or metastatic tumor with a calculated TMB-high, defined as $\geq 10 \mathrm{mut} / \mathrm{Mb}$ for solid tumors. $^{9}$

TMB is not the only factor predictive of response to immunotherapy, additional factors include the ability of tumor-infiltrating lymphocytes (TILs) to invade the tumor and other immune-related factors in the tumor microenvironment, she reminded attendees.

Tissue supply can be another obstacle in using TMB, but one that might be ameliorated with the use of ctDNA. A recent study used ctDNA to calculate TMB in 52 patients with advanced NSCLC and demonstrated a good correlation with outcomes related to pembrolizumab. ${ }^{10}$ Patients with a high TMB $(\geq 16$ mutations $/ \mathrm{mb})$ had a $70 \%$ reduced risk of disease progression $(P<.001)$ and a $52 \%$ reduced risk of dying $(P=.061)$, relative to patients with a lower TMB.

Efforts are currently underway to validate additional immunotherapy markers and streamline their testing.

Disclosures: Dr. Morrissette has disclosed that she has received consulting fees from Novartis Pharmaceuticals Corporation and BioRad.

Correspondence: Jennifer J.D. Morrissette, PhD, University of Pennsylvania, 3020 Market Street, Suite 220, Philadelphia, PA 19104.

Email: jemorris@pennmedicine.upenn.edu

\section{References}

1. Association for Molecular Pathology Training and Education Committee. ONCOLOGY: Interpretation of Genomic Assays. Revised October 2019. Accessed July 5, 2020. Available at: https://www.amp.org/AMP/assets/ File/education/MIMP/NGSInterpretation_10_8_19.pdf?pass=15

2. Zehir A, Benayed R, Shah RH, et al. Mutational landscape of metastatic cancer revealed from prospective clinical sequencing of 10,000 patients [published correction appears in Nat Med 2017;23:1004]. Nat Med 2017; 23:703-713

3. Zill OA, Banks KC, Fairclough SR, et al. The landscape of actionable genomic alterations in cell-free circulating tumor DNA from 21,807 advanced cancer patients. Clin Can Res 2018;24:3528-3538.

4. Zhang Y, Chang L, Yang Y, et al. Intratumor heterogeneity comparison among different subtypes of non-small-cell lung cancer through multiregion tissue and matched ctDNA sequencing. Mol Cancer 2019;18:7.

5. Aggarwal C, Thompson JC, Black TA, et al. Clinical implications of plasma-based genotyping with the delivery of personalized therapy in metastatic non-small cell lung cancer. JAMA Oncol 2019;5:173-180.
6. Mittmann N, Earle CC, Cheng SY, et al. Population-based study to determine the health system costs of using the 21 -gene assay. J Clin Oncol 2018;36:238-243.

7. Chalmers ZR, Connelly CF, Fabrizio D, et al. Analysis of 100,000 human cancer genomes reveals the landscape of tumor mutational burden. Genome Medicine 2017;9:34.

8. Buchhalter I, Rempel E, Endris V, et al. Size matters: dissecting key parameters for panel-based tumor mutational burden analysis. Int J Cancer 2019;144:848-858.

9. US Food \& Drug Administration. FDA approves pembrolizumab for adults and children with TMB-H solid tumors. Accessed June 5, 2020. Available at: https://www.fda.gov/drugs/drug-approvals-and-databases/fda-approves-pembrolizumab-adults-and-children-tmb-h-solid-tumors.

10. Aggarwal C, Thompson JC, Chien AL, et al. Baseline plasma tumor mutation burden predicts response to pembrolizumab-based therapy in patients with metastatic non-small cell lung cancer. Clin Cancer Res 2020; 26:2354-2361. 